\title{
CARACTERÍSTICAS HISTOPATOLÓGICAS PARA EL DESARROLLO DE CÁNCER GÁSTRICO EN PACIENTES CON DISPEPSIA
}

\author{
HISTOPATHOLOGICAL CHARACTERISTICS FOR DEVELOPMENT OF GASTRIC CANCER
}

IN PATIENTS WITH DYSPEPSIA

\author{
CARATERISTICAS HISTOPATOLÓGICAS PARA O DESENVOLVIMENTO DO CANCER \\ GASTRICO EM PACIENTES COM DISPEPSIA
}

\author{
VIRGILIO ALVARADO GALLO', LINDA NARANJO RAFFO² \\ ${ }^{1}$ Hospital Universitario de Guayaquil, Guayaquil, Ecuador \\ 2 Universidad de Guayaquil, Guayaquil, Ecuador
}

Introducción: en la patogénesis del cáncer gástrico el único método de diagnóstico es el estudio histopatológico que suele realizarse en etapas tardías. La detección oportuna de las lesiones preneoplásicas, disminuirían los cambios irreversibles de la mucosa gástrica y a su vez la incidencia de su malignidad. Objetivos: determinar las características histopatológicas preneoplásicas para el desarrollo de cáncer gástrico y su asociación con la edad y el sexo. Materiales y métodos: se realizó un estudio observacional, transversal y descriptivo donde se incluyó 1025 endoscopías digestivas con resultados histopatológicos en el área de gastroenterología del hospital Universitario de Guayaquil, periodo 2014-2015. Resultados: de los 1025 casos, el $80.8 \%$ ya tenían cambio en la mucosa gástrica representada por signos histológicos crónicos, cuyos síntomas inespecíficos se presentaban en un $79 \%$; el $17.3 \%$ presentaron signos preneoplásicos cuyos síntomas inespecíficos ocurrieron en un $75.8 \%$. El principal motivo de estudio en todas las lesiones histológicas fue la dispepsia. Conclusiones: los síntomas inespecíficos tuvieron un alto porcentaje de sospecha para indicar la realización de una endoscopía digestiva alta, de ahí la importancia de optimizar la historia clínica, en busca de síntomas precoces, factores de riesgo que contribuyan al desarrollo de neoplasia gástrica. PALABRAS CLAVE: patología, dispepsia, metaplasia, neoplasias gástricas.

Abstract

INTRODUCTION: in the pathogenesis of gastric cancer, the diagnosis method is the histopathological study, usually done in later stages. Timely detection of preneoplastic lesions would diminish irreversible changes in the gastric mucosa and, in turn, the incidence of malignancy. Objectives: to determine the histopathological preneoplastic characteristics for development of gastric cancer and its association with age and sex. Materials and methods: an observational, cross-sectional and descriptive study was conducted, which included 1025 digestive endoscopies with histopathological results in the gastroenterology area of the hospital Universitario de Guayaquil (University Hospital of Guayaquil) within the period 2014-2015. Results: out of the 1025 cases, $80.8 \%$ already showed a change in the gastric mucosa represented by chronic histological signs, for which nonspecific symptoms were present in $79 \%$ of the cases; $17.3 \%$ showed preneoplastic signs with nonspecific symptoms in $75.8 \%$. The main cause for all histological lesions under study was dyspepsia. Conclusions: nonspecific symptoms had a high percentage of suspicion to suggest the performance of an upper digestive endoscopy, hence the importance of optimizing the clinical history, so as to find early symptoms, risk factors that contribute to the development of gastric neoplasia. KEYWORDS: pathology, dyspepsia, metaplasia, stomach neoplasms.

Resumo

INTRODUÇÃo: na patogênese do câncer gástrico o único método de diagnóstico é a histopatologia o que geralmente ocorre em estágios tardios. A detecção precoce de lesões pré-malignas, diminuirão as alterações irreversíveis da mucosa gástrica e, por sua vez, a incidência de malignidade. Objetivos: identificar características histopatológicas pré-malignas para o desenvolvimento de câncer gástrico e sua associação com a idade e sexo. Materiais e Métodos: é realizado 0 estudo observacional, transversal e descritivo de 1025 endoscopias com achados histopatológicos incluídos na área de gastroenterologia do Hospital Universitário de Guayaquil, no período de 2014 a 2015. Resultados: dos 1025 casos, 0 80,8 \% já tinham alterações na mucosa gástrica representada por sinais histológicos crônicos, cujos sintomas inespecíficos se apresentaban num 79 \%; 0 17,3 \% apresentaram sinais pré-malignas cujos sintomas inespecíficos foram apresentados num 75,8\%. A principal razão para o estudo em todas as lesões histológicas foi dispepsia. Conclusões: sintomas inespecíficos tinha uma elevada percentagem de suspeita para indicar a realização de uma endoscopia digestiva alta, daí a importância de otimizar a história médica, procurando os primeiros sintomas, fatores de risco que contribuem para o desenvolvimento de câncer gástrico.

PALABRAS-CHAVE: patologia, dispepsia, metaplasia, neoplasias gástricas. 


\section{INTRODUCCIÓN}

La patogénesis del cáncer gástrico es un proceso en que intervienen múltiples factores como son la predisposición genética, la dieta, los factores ambientales, la infección por Helicobacter pylori, entre otros, que influyen en el desarrollo secuencial de la neoplasia gástrica. ${ }^{1}$ En la historia natural del adenocarcinoma gástrico, cada vez toma mayor fuerza como factor precursor la gastritis crónica no atrófica, gastritis crónica atrófica, metaplasia intestinal, displasia y el adenocarcinoma gástrico. ${ }^{2}$ La biopsia gástrica es el único método diagnóstico que permite conocer el tipo, la intensidad y la extensión de estas alteraciones.

En nuestro medio el diagnóstico se lo realiza en etapa tardía por la cual la supervivencia de los pacientes es pobre, esto puede estar también influido por los hábitos alimentarios (ahumados, salados, nitrosaminados), tabaco, alcohol ${ }^{3}$ y la falta de información sobre los síntomas inespecíficos (dolor en el hemiabdomen superior, náuseas, vómitos, dispepsia) lo que conlleva a que el paciente se automedique para aliviar los síntomas y prolongue la visita al médico en etapas tempranas. La dispepsia constituye el síntoma inespecífico más frecuente de consulta al especialista; $y$ siguiendo las recomendaciones científicas el médico de atención primaria y el no especialista deberían utilizar el tratamiento erradicador empírico. ${ }^{4}$

El seguimiento de las lesiones preneoplásicas difiere de un paciente a otro dependiendo de la etnia, país, antecedentes familiares, factores de riesgo, y del tipo histológico/extensión encontrado. Las características histopatológicas preneoplásicas para el desarrollo de cáncer gástrico cada vez toman mayor importancia para prevenir y detectar, de manera oportuna, cambios en la mucosa gástrica que pueden ser reversibles e irreversibles en la secuencia evolutiva de un cáncer gástrico. La endoscopía digestiva junto con las nuevas técnicas y el juicio clínico constituyen el diagnóstico definitivo para estas lesiones.

El propósito del presente estudio es detectar las características histopatológicas preneoplásicas como la atrofia, metaplasia intestinal y la displasia como precursores del desarrollo del cáncer gástrico en el contexto de diversa sintomatología gastrointestinal. Además establecer la edad y el sexo más frecuente en que se encuentran estos cambios histopatológicos gástricos, con el fin de orientar a los médicos de atención primaria y a los no especialistas respecto a los grupos de riesgo.

\section{MATERIALES Y MÉTODOS}

Se desarrolló un estudio transversal, observacional y descriptivo. Se analizaron todas las endoscopías altas en la que se realizó biopsia de los diferentes segmentos de la mucosa gástrica en 1025 endoscopías realizadas en la unidad de gastroenterología del hospital Universitario de Guayaquil de enero de 2014 a enero de 2015.

Se analizaron los resultados histopatológicos tomando como base de datos, los reportes de patología y estadística de la institución, ubicadas en los archivos de historias clínicas donde se evaluaron las características histológicas y el motivo de estudio; es decir, la sintomatología que llevóal especialista a realizar el procedimiento. Se agruparon los pacientes de acuerdo al resultado histopatológico en: signos histológicos de cronicidad (gastritis crónica, pólipos hiperplásicos y pólipos adenomatosos), con signos histológicos preneoplásicos (atrofia gástrica, metaplasia intestinal y displasia gástrica) y signos neoplásicos (adenocarcinoma gástrico). También se los agrupó de acuerdo a la sintomatología en: síntomas inespecíficos (dispepsia, reflujo, cirrosis hepática, cuerpo extraño, controles de lesiones premalignas y acromegalia) y síntomas de alarma (hemorragia digestiva, pérdida de peso, disfagia, anemia y masa abdominal).

Para el análisis estadístico descriptivo, se utilizaron las variables cualitativas frecuencia y porcentaje. Para el análisis inferencial, se calculó el Chi cuadrado para valorar la asociación entre las variables. El software empleado para el análisis fue Microsoft Excel ${ }^{\circledR}$.

Los procedimientos realizados para la recolección de datos y análisis de los mismos se siñeron a las normas de ética de acuerdo a la declaración de Helsinki; se protegió y se mantuvo en reserva la información personal de los participantes.

\section{RESULTADOS}

De las 1025 video endoscopías digestivas altas con biopsias se obtuvieron: con signos histológicos de cronicidad 828 pacientes $(80.8 \%): 806$ con gastritis crónica, 19 con pólipos hiperplásicos y 3 con pólipos adenomatosos, con signos histológicos preneoplásicos 178 (17.3\%) pacientes: 15 con atrofia gástrica, 159 con metaplasia 
intestinal, 4 con displasia gástrica. Finalmente, con signos neoplásicos (adenocarcinomas) se observó $19(1.9 \%)$ pacientes (Tabla 1$)$.

\section{TABLA 1. INDICADORES HISTOPATOLÓGICOS GÁSTRICOS}

\begin{tabular}{lrr}
\hline SIGNOS HISTOPATOLÓGICOS & $\mathbf{N}^{\circ}$ & \% \\
\hline De cronicidad & 828 & 80.8 \\
\hline Preneoplásicos & 178 & 17.3 \\
\hline Neoplásicos & 19 & 1.9 \\
\hline Total & 1025 & 100
\end{tabular}

Se encontró una diferencia estadísticamente significativa (valor $\mathrm{p}<0,001$ ) donde los pacientes con histopatología neoplásica presentaban mayor cantidad de resultados indiferente al $\mathrm{H}$. pylori (Tabla 2).

\begin{tabular}{|c|c|c|c|c|}
\hline $\begin{array}{l}\text { SIGNOS } \\
\text { HISTOPATOLÓGICOS }\end{array}$ & SI & NO & INDIFERENTE & TOTAL \\
\hline De cronicidad & $\begin{array}{r}672 \\
(81,1 \%)\end{array}$ & $\begin{array}{r}148 \\
(17,9 \%)\end{array}$ & $8(1 \%)$ & 828 \\
\hline Preneoplásicos & $\begin{array}{r}160 \\
(89,9 \%)\end{array}$ & $17(9,5 \%)$ & $1(0,5 \%)$ & 178 \\
\hline Neoplásicos & $3(15,8 \%)$ & $2(10,5 \%)$ & $14(73,7 \%)$ & 19 \\
\hline Total & 835 & 167 & 23 & 1025 \\
\hline
\end{tabular}

La edad media de presentación en el grupo de cambios histológicos gástricos crónicos fue de 48.7 años; la edad media del grupo de indicadores histológicos preneoplásicos fue de 60.3 años; y la edad media de presentación de aquellos con signos histológicos neoplásicos gástricos fue 61.4 años.

De acuerdo a la sintomatología de los 828 pacientes con signos histológicos crónicos, 654 (79\%) presentaron síntomas inespecíficos; 556 dispepsia; 44 reflujo; 26 control de lesiones preneoplásicas; 12 acromegalia; 11 cirrosis hepática; y, 5 cuerpo extraño; 174 (21\%) presentaron síntomas de alarma; 136 hemorragia digestiva; 16 disfagia; 13, pérdida de peso y 9 anemia. De los 178 pacientes con signos histológicos preneoplásicos, 135 (75.8 \%) tuvieron síntomas inespecíficos; 112 dispepsia; 12 control por lesiones preneoplásicas; 5 reflujo; 3 , cirrosis hepática; 3, cuerpo extraño, y 43 (24.2\%) pacientes presentaron síntomas de alarma; 33 hemorragia digestiva; 5 anemia; 4 disfagia, 1 pérdida de peso. De los 19 pacientes con signos neoplásicos, 4 (21.1\%) síntomas inespecíficos (dispepsia) y 15 (78.9 \%) síntomas de alarma; 7 masa abdominal, 4 hemorragia digestiva, 2 anemia, la pérdida de peso, 1 disfagia. (Figura 1).

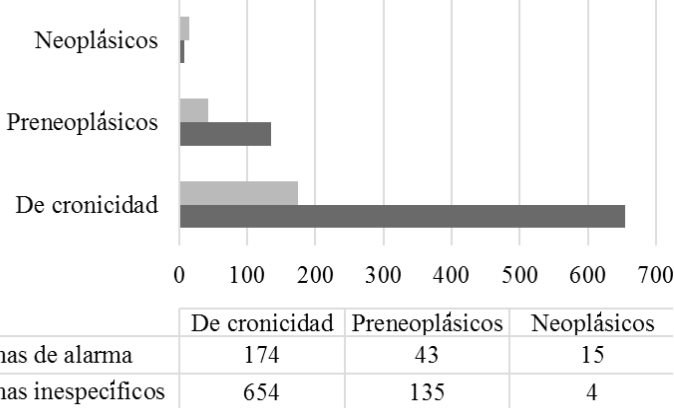

Figura 1. Indicadores histopatológicos en el contexto de sintomatología gastrointestinal.

El sexo femenino fue el más frecuente en todos los indicadores histológicos gástricos en relación con el sexo masculino. En cuanto a signos histológicos de cronicidad, el $68.4 \%$ fueron mujeres y el $31.6 \%$ hombres; con signos histológicos preneoplásicos el 59.6\% fue de sexo femenino y el $40.4 \%$ sexo masculino; y, en los que tuvieron signos histológicos neoplásicos, el $57.9 \%$ fueron mujeres y el $42.1 \%$ hombres. No se encontró que esta asociación fuera estadísticamente significativa (valor p 0,077). (Tabla 3).

TABLA 3. INDICADORES HISTOPATOLÓGICOS EN RELACIÓN CON EL SEXO

\begin{tabular}{lrrr}
$\begin{array}{l}\text { Signos } \\
\text { HISTOPATOLógIcos }\end{array}$ & SEXO FEMENINO & SEXO MASCULINO & TOTAL \\
\hline De cronicidad & $551(68,4 \%$ & $255(31,6 \%)$ & 806 \\
\hline Preneoplásicos & $121(60,5 \%)$ & $79(39,5 \%)$ & 200 \\
\hline Neoplásicos & $11(57,9 \%)$ & $8(42,1 \%)$ & 19 \\
\hline Total & 683 & 342 & 1025 \\
\hline
\end{tabular}

Prueba de Chi cuadrado $=5.478$

\section{DISCUSIÓN}

El presente trabajo representa para el hospital Universitario un acercamiento para determinar histológicamente las lesiones premalignas gástricas evaluadas en el área de gastroenterología. El adenocarcinoma gástrico representa, a nivel mundial uno de los cánceres con mayor tasa de mortalidad, ocupando el tercer lugar según la OMS en el año 2012. ${ }^{5}$ Según la GLOBOCAN $2012^{6}$ en Ecuador la mortalidad por cáncer gástrico en hombres ocupa 
el segundo lugar con un $19 \%$ precedido del cáncer de próstata; en mujeres ocupa el tercer lugar con un $13.8 \%$ precedido del cáncer de cuello uterino y de mama. ${ }^{6,7}$

No existe estadística a nivel nacional sobre lo que representa las lesiones preneoplásicas gástricas que constituyen uno de los factores de riesgo más importantes en la génesis del adenocarcinoma gástrico, teniendo en cuenta que en Ecuador, según el registro nacional de tumores en el año 2013 (publicado el 16 de abril de 2015), indica que se han presentado 1437 casos de cáncer de estómago en hombres, con una tasa de 18.4 por cada 100.000 habitantes y en mujeres, 917 casos con una tasa de 11.5 por cada 100.000 habitantes; ${ }^{7}$ no cabe duda que el cáncer gástrico constituye un problema de salud pública.

Existen varios consensos acerca de la evolución del adenocarcinoma gástrico; la gran mayoría tienen una característica en común, en que son precedidos por una secuencia de cambios histológicos que incluyen: gastritis crónica, atrofia gástrica, metaplasia intestinal, displasia gástrica y según su intensidad y persistencia, incrementan el riesgo de desarrollar cáncer gástrico; ${ }^{8}$ es por eso que el enfoque del presente trabajo de investigación, está dirigido a lo que precede el adenocarcinoma gástrico, es decir, a los signos histológicos preneoplásicos y su relación con los síntomas inespecíficos y de alarma que consulta el paciente, sin olvidar la estrecha relación con la infección del helicobacter pylori. Aún cuando no se ha podido demostrar la causa y el efecto ${ }^{9}$, la International Agency for Research of Cancer (IARC) ${ }^{10} \mathrm{y}$ la OMS lo categorizaron como agente carcinogénico tipo I.

Existen varios estudios en cuanto a la relación de las lesiones preneoplásicas con el helicobacter pylori, como para el desarrollo posterior a adenocarcinoma gástrico, y comparándolos con este estudio, no existe mayor diferencia, teniendo en cuenta los diferentes métodos utilizados en la investigación.

Por ejemplo, en Perú se realizó un estudio sobre las lesiones gástricas preneoplásicas y helicobacter pylori en despistaje endoscópico para cáncer gástrico, en población de nivel socioeconómico medio y alto. ${ }^{11}$ Lo interesante es que siendo una población de estudio de 2616, tuvieron una prevalencia baja de lesiones premalignas representada por un $7.1 \%$ pero con una fuerte relación con el helicobacter pylori. También realizaron otro estudio interesante so- bre los factores de riesgo asociados a metaplasia intestinal gástrica en pacientes sin enfermedad gastroduodenal significativa en la que dudan sobre la asociación de la infección por helicobacter pylori, y concluyen que la presencia de reflujo biliar y el antecedente de consumo de alcohol en la población estudiada, representan los factores de riesgo para el desarrollo de una lesión premaligna como lo es la metaplasia intestinal; la infección por helicobacter pylori no se comportó como un factor de riesgo. ${ }^{12}$

En Argentina y en Colombia se realizaron estudios sobre la utilidad de la cromoendoscopía en la detección de lesiones gástricas premalignas y malignas; ambos concluyeron sobre la importancia de este método en cuanto a dirigir las biopsias en una mucosa con alta sospecha de malignidad mediante la tinción con azul de metileno. ${ }^{13,14}$

En Colombia realizaron un estudio similar al presente, basado en las lesiones precursoras de malignidad (gastritis crónica atrófica, metaplasia intestinal y displasia leve); el referido estudio fue prospectivo, descriptivo, con 212 pacientes entre 11 y 89 años de edad; la prevalencia para gastritis crónica atrófica antro corporal fue de 38,6 \%, metaplasia intestinal 24,4\% y displasia leve 1,5\%; presencia de infección para helicobacter pylori en gastritis crónica atrófica 73,5\%, para metaplasia intestinal $52 \%$ y displasia leve $100 \%$; en relación a la severidad de las lesiones precursoras de malignidad de acuerdo a la escala de OLGA, 11,5\% se clasificó como estadios III y IV; a excepción de un solo caso clínico, todos fueron helicobacter pylori positivos. ${ }^{15}$

El seguimiento de los indicadores premalignos según un estudio realizado en EE.UU indica que la metaplasia intestinal a menudo crea incertidumbre en el manejo de la misma ya que el riesgo de cáncer gástrico aumenta con su presencia, si bien es cierto el cáncer gástrico en Estados Unidos y en otros países occidentales es de menor incidencia, en relación con Asia y América Latina, por ende es difícil elaborar programas óptimos de vigilancia. ${ }^{16}$

Autores sin embargo sugieren que la vigilancia de la metaplasia intestinal difiere en diferentes aspectos: origen étnico, ubicación geográfica, historia familiar, factores de riesgo, el juicio clínico individual y en cuanto a la lesión según el tipo histológico y su grado de extensión. La atrofia casi siempre se relaciona con la metapla- 
sia intestinal, estas lesiones por sí solas no constituyen un indicador de vigilancia endoscópica prolongada a menos que existan otros factores de riesgo para el cáncer gástrico. ${ }^{16}$

La displasia de bajo grado tiene su controversia, unos sugieren vigilancia endoscópica anual con rebiopsia y otros sugieren vigilancia con cromatografía cada tres meses con biopsia por lo menos durante el primer año y suspenderla cuando dos endoscopias consecutivas sean negativas. La displasia de alto grado debe ser confirmada por dos patólogos y su inmediata resección quirúrgica o endoscópica. ${ }^{16}$

Todos estos indicadores preneoplásicas deben ser seguidos de preferencia por nuevos métodos tecnológicos como es la cromatoendoscopia para evaluar su extensión y descartar lesiones más avanzadas y neoplasias en estadio temprano, teniendo presente que la determinación de los niveles de pepsinógeno también son un buen marcador para valorar la atrofia gástrica. ${ }^{17}$

Haciendo referencia al estudio realizado en el área de gastroenterología del hospital universitario, cada cuatro pacientes con signos histológicos gástricos crónicos, uno desarrollará signos histológicos preneoplásicos, pudiendo ser atrofia gástrica, metaplasia intestinal o displasia gástrica; y cada nueve pacientes con signos histológicos preneoplásicos, uno evolucionará a adenocarcinoma gástrico según el presente estudio. Sobre los indicadores histológicos en el desarrollo de cáncer gástrico en relación con la sintomatología, se considera que cada cuatro pacientes con síntomas inespecíficos uno tendrá como resultado histopatológico un indicador preneoplásico, y de cada doce pacientes con síntomas de alarma, uno tendrá como resultado histopatológico lesión neoplásicas.

\section{CONCLUSIONES}

Los síntomas que motivaron a realizarse una videoendoscopía digestiva alta fueron en un alto porcentaje síntomas inespecíficos y no de alarma, excepto en los pacientes que presentaron adenocarcinoma gástrico; de ahí la importancia de optimizar la historia clínica, en busca de síntomas precoces, antecedentes familiares, y factores de riesgo que contribuyan al desarrollo de neoplasia gástrica, así como también el buen criterio del profesional para seleccionar quienes deberían realizarse video endoscopía digestiva alta siguiendo los patrones conceptuados en las diferentes guías clínicas. El 17.3 \% de los pacien- tes que presentaron indicadores preneoplásicos ¿cuántos de ellos desarrollaran cáncer gástrico en un futuro?. El diagnóstico precoz de lesiones pre neoplásicas se potenciaría con el uso de nuevas técnicas como es la video endoscopía con cromatografía ${ }^{13}$, sobre todo en países donde existe alta prevalencia de la infección con helicobacter pylori.

\section{REFERENCIAS BIBLIOGRÁFICAS}

1. Gisbert J, Calvet X, Bermejo F, Boixeda D, Bory F, Bujanda L, et al. III Conferencia Española de consenso sobre la infección por Helicobacter pylori. Elsevier. 2013; 36: 340-74.

2. Correa P, Haenszel W, Cuello C, Zavala D, Fontham E, Zamora G, et al. Gastric precancerous process in a high risk population: cohort follow up. Pubmed. 1990; 50 (15): 4737-40.

3. Sopeña F, Garcia M. Prevención primaria del Cáncer gástrico. Elsevier. 2011; 10(4): 163-9.

4. Ferrández Á, Campillo A, Bernal V. Dispepsia funcional y orgánica. Manejo general y extrahospitalario del paciente con dispepsia no investigada. Medicine. 2008; 10(2): 101-7.

5. OMS $\mid$ Cáncer [Internet]. WHO. World Health Organization; 2015 [cited 2017 Feb 7]. Available from: http://www.who. int/mediacentre/factsheets/fs297/es/

6. WHO. Estimated Cancer Incidence Mortality and Prevalence Worldwide 2012 [Internet]. World Health Organization. 2012 [cited 2017 Feb 7]. Available from:http:// globocan.iarc.fr/Pages/fact_sheets_cancer.aspx.

7. Salud SA. El cáncer en el Ecuador. Salud total. Cueva P. 2015 [actualización abril de 2015; acceso diciembre de 2015]. Disponible en: salud.sa.com/ vinesaludtotal/ index.php/cáncer/elcancer-en-el- ecuador/423.el-canceren-el-ecuador.

8. Piñol F, Salvador J, Paniagua M, Borbolla E. Características de la infección por Helicobacter pylori en pacientes con adenocarcinoma gástrico. Rev cubana med. 2005; 44(5-6): 1-15.

9. Rollán A, Cortés P, Calvo A, Araya R, Bufadel M, González $\mathrm{R}$, et al. Diagnóstico precoz de cáncer gástrico. Propuesta de detección y seguimiento de lesiones premalignas gástricas: protocolo ACHED. Rev Med Chile. 2014; 42: 1181-1192.

10. OPS. Epidemiología del Cáncer de estómago en las Américas, 2014. Francia. Iarc. 2012 [actualización 2014; acceso diciembre 2015]. Disponible en: http://www. paho.org/hq/index.php?option=com_docman\&task=doc_ view\&gid=21596\&Itemid $=270$.

11. Chacaltana A, Rodríguez C, Urday C, Ramon W, Espinoza $\mathrm{J}$, Velarde $\mathrm{H}$, et al. Lesiones gástricas preneoplásicas y helicobacter pylori en despistaje endoscópico para cáncer gástrico en población de nivel socioeconómico medio y alto. Rev. Gastroenterol. Perú. 2009; 29(3): 218-225.

12. Chacaltana A, Soriano C, Frisancho O. Factores de Riesgo Asociados a Metaplasia Intestinal Gástrica en Pacientes 
sin Enfermedad Gastroduodenal Significativa. ¿Está Siempre Asociada la Infección por Helicobacter Pylori? Rev. Gastroenterol. Peru. 2012; 32(1): 50-57.

13. Calle Astudillo G, Jerves T, Pesántez L, Calle P, Gutiérrez A, Calle G. Utilidad de las biopsias gástricas rutinarias y de la tinción con azul de metileno en el diagnóstico de la metaplasia intestinal en mayores de 40 años. Acta Gastroenterol Latinoam. 2013; 43: 189-197.

14. Emura F, Mejía J, Mejía M, Osorio C, Hernández C, González I, et al. Utilidad de la cromoendoscopia sistemática en el diagnóstico del cáncer temprano y lesiones gástricas premalignas. Resultado de dos campañas masivas consecutivas de tamización en Colombia (2006-2007). Rev Col Gastroenterol. 2010; 25-1: pagina 19-30..

15. Bedoya A, Sansón F, Yépez J, Fuertes V, Santacruz C, Cifuentes Y, et al. Prevalencia y severidad de las lesiones precursoras de malignidad en una área de alto riesgo de cáncer gástrico. Pasto 2012. Rev Col Gastroenterol 2012; 27 (4): 276-281.

16. Correa P, Piazuelo M, Wilson K. Pathology of Gastric Intestinal Metaplasia: Clinical Implications. Am J Gastroenterol. 2010; 105(3): 493-498.

17. Gómez Zuleta M, Riveros J, Ruiz O, Concha A, Ángel D, Torres $M$, et al. Guía de práctica clínica para la prevención, diagnóstico y tratamiento del cáncer gástrico temprano-2015. Rev Col Gastroenterol. 2015. 30(1): 34-42. 